\title{
Reconstrucción auricular en lesiones malignas de hélix
}

\author{
Auricle reconstruction in malignant helix lesions
}

Maximiliano Oscar Ábalos', Belén Bruno Cherot², Nathaniel Dionisio Arellanos ${ }^{3}$, Justo La Torre Viscarra ${ }^{4}$

\section{RESUMEN}

La complejidad del pabellón auricular hace que su reconstrucción sea técnicamente dificultosa. Hay una gran variedad de técnicas para la reconstrucción del hélix luego de la resección tumoral. La cirugía de elección en los centros especializados es la de Mohs; sin embargo, no se dispone de ella en todos los centros, por lo que debemos tener claras las indicaciones de las técnicas tradicionales. Como objetivo evaluamos la resolución quirúrgica de lesiones malignas ubicadas en el hélix auricular y el resultado estético de acuerdo al tamaño tumoral y su localización. Realizamos un análisis retrospectivo de 7 pacientes tratados con la técnica de cuña escalonada y la de Antia y Buch para defectos de hasta $3 \mathrm{~cm}$ del hélix auricular. Concluimos que ambas tienen una extraordinaria versatilidad y un excelente resultado estético funcional, y que la elección de una u otra se basará en el tamaño auricular y el excedente de piel que tengamos al momento de la reconstrucción.

Palabras claves: reconstrucción auricular, hélix, lesiones malignas, cuña, Antia.

\begin{abstract}
The complexity of the earpiece makes its reconstruction technically complicated. There are several techniques for reconstruction of the helix after tumor resection. Gold standard in specialized centers is Mohs's surgery; however, you do not have it at all centers, so we must have clear indications of traditional techniques. The aim is to evaluate the surgery resolution of malignant pathology in the auricular helix and the aesthetic result according to tumor size and location. We describe a retrospective analysis of 7 patients treated with the technique step wedge and Antia and Buch for defects up to $3 \mathrm{~cm}$ earpiece helix. We conclude that both have an extraordinarily functional versatility with excellent cosmetics result, and we think in choosing one or the other based on the ear size and excess of skin that we have at the time of reconstruction.
\end{abstract}

Keywords: auricular reconstruction, helix, malignant injuries, wedge, antia.

\section{INTRODUCCIÓN}

La complejidad del pabellón auricular hace que su reconstrucción sea técnicamente dificultosa, debido a las concavidades y convexidades del cartílago subyacente con su adherente y delgada capa de piel que lo recubre. El porcentaje de resecciones para los carcinomas basocelulares en oreja es de un $14,5 \%$, y el $17 \%$ de estos corresponde a la región del hélix ${ }^{1}$.

En el caso de los espinocelulares, el porcentaje es de $7,5 \%$ y de estos un $20,5 \%$ son del hélix auricular ${ }^{1}$.

Hay una gran variedad de técnicas para la reconstrucción del hélix luego de la resección tumoral. Durante los últimos 20 años ha sido de elección la cirugía micrográfica de Mohs, ya que preserva la mayor cantidad de tejido libre de lesión lo que conlleva a un mejor resultado no solo oncológico sino también estético-funcional ${ }^{4}$.

1. Cirujano plástico y reconstructivo. Director médico de Exea Salud.

2. Instructora de Residentes del Servicio de Cirugía Plástica del Hospital Tornú.

3. Médico de planta del Servicio de Cirugía Plástica del Hospital Tornú, Buenos Aires.

4. Jefe del Departamento de Cirugía del Hospital Tornú, Buenos Aires. Miembro Titular SACPER.

$\square$ Correspondencia: Maximiliano Ábalos. Tel: 1164348302. maximilianoabalos@gmail.com

Los autores no declaran conflictos de intereses

Recibido: 02/02/2020 / Aceptado: 10/02/2020
Nuestro objetivo es evaluar la resolución quirúrgica de lesiones malignas ubicadas en el hélix auricular y el resultado estético de acuerdo al tamaño tumoral y su localización.

\section{MATERIALES Y MÉTODOS}

Se realizó un análisis retrospectivo entre los años 2012 y 2016 del Servicio de Cirugía Plástica del Hospital General de Agudos Dr. Enrique Tornú, seleccionando los pacientes que fueron intervenidos quirúrgicamente por presentar lesiones malignas y premalignas en el hélix auricular.

\section{CRITERIOS DE INCLUSIÓN}

Pacientes intervenidos quirúrgicamente con seguimiento en el Hospital General de Agudos Dr. E. Tornú.

Lesiones malignas y premalignas ubicadas en el hélix auricular.

Resolución quirúrgica mediante la técnica de cuña escalonada y Antia y Buch.

\section{CRITERIOS DE EXCLUSIÓN:}

Lesiones benignas.

Resolución quirúrgica mediante otra técnica.

Localización auricular fuera del hélix.

Se presentan 7 pacientes que cumplían los criterios de inclusión, con un rango etario de 60 a 91 años de edad (media 69 años), seis de sexo masculino. 

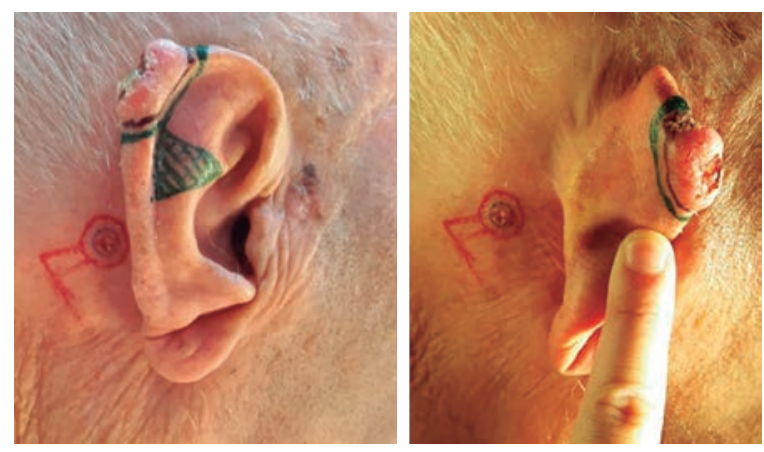

Figura 1. Marcación de la cuña escalonada.

Cuatro de ellos tenían diagnóstico histológico de epitelioma basocelular (EBC), tres diagnóstico de epitelioma espinocelular (EEC) y uno de queratoacantoma. Cuatro fueron tratados con la técnica de cuña escalonada, y tres con colgajo de Antia y Buch.

Los márgenes de seguridad que se utilizaron fueron de $5 \mathrm{~mm}$ para los EBC, $6 \mathrm{~mm}$ para los EEC y $3 \mathrm{~mm}$ para el queratoacantoma.

Los defectos a resecar que fueron seleccionados para la reconstrucción mediante la técnica de cuña escalonada medían hasta $2,5 \mathrm{~cm}$, incluyendo el margen de seguridad.

A quienes se les realizó Antia y Buch, medían hasta 3,6 $\mathrm{cm}$ el mayor con sus márgenes de seguridad incluidos.

A todos se les solicitó laboratorio prequirúrgico, riesgo cardiológico y firmaron consentimiento informado. Todas las cirugías se realizaron con anestesia local. La analgesia posoperatoria se instauró con diclofenac reglado cada 12 hs y se les indicó antibioticoprofilaxis preoperatoria.

El seguimiento posoperatorio se realizó cada 48 hs la primera semana, luego a los 7 y 15 días, posteriormente a 1, 2, 6 y 12 meses.

\section{RESULTADOS}

\section{TÉCNICA DE CUÑA ESCALONADA ${ }^{4}$}

Cumpliendo con los criterios oncológicos pertinentes, el defecto quirúrgico se convierte en un rectángulo de grosor completo. El ancho del defecto, correspondiente topográficamente con la base de la lesión, nos dará una longitud determinada que denominaremos segmento $\mathrm{AB}$. A continuación, se confecciona un triángulo de compensación que abarque las regiones del antihélix y la concha, craneal o caudalmente al defecto según sea el caso. La base de este triángulo, se denominará segmento $\mathrm{A} 1 \mathrm{~B} 1$ y su longitud será igual a la longitud del segmento $\mathrm{AB}$. El triángulo tiene que ser isósceles y la bisectriz del ángulo del vértice tiene que ser orientada hacia un punto localizado en la raíz del hélix. Este triángulo se extirpa en todo su espesor (Figura 1). Los bordes del hélix y antihélix son avanzados y se suturan, reestableciendo la continuidad anatómica condrocutánea y dando como resultado una línea de sutura en Z (Figura 2).
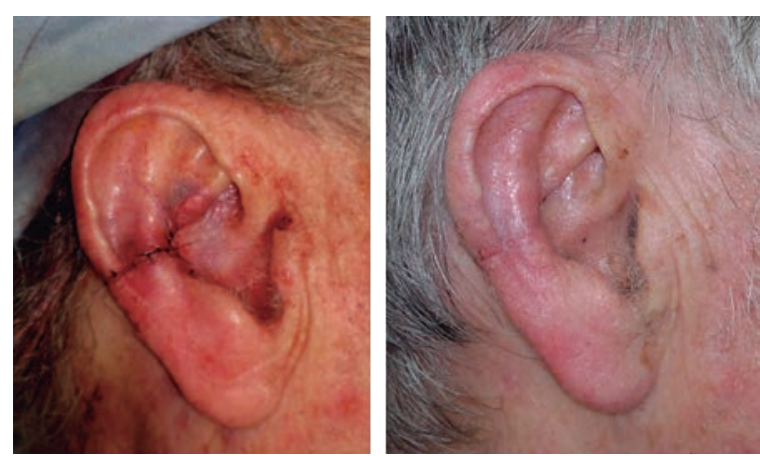

Figura 2. Posoperatorio mediato y a los 6 meses.

\section{TÉCNICA DE ANTIA Y BUCH²}

Luego de la resección oncológica con los márgenes de seguridad correspondientes de acuerdo a cada caso, se realiza en primera instancia la liberación total del hélix desde la escafa, a través de una incisión realizada sobre el surco el hélix y que abarca todo el espesor auricular. En segundo lugar, se diseca la piel de la zona posterointerna de la oreja, avanzando por encima del pericondrio, hasta que todo el hélix quede suspendido como un componente condrocutáneo de la piel laxa movilizada. Se puede ganar longitud adicional mediante un avance en V-Y del pilar del hélix y de esta forma cerrar grandes defectos sin tensión (Figura 3).

No se presentaron complicaciones en los pacientes. El resultado estético funcional fue satisfactorio, no hubo cicatrización patológica, tampoco recidivas, todos los márgenes se informaron libres de lesión y toleraron bien la cirugía bajo anestesia local.

\section{DISCUSIÓN}

Existen muchas opciones de reconstrucción del hélix luego de la resección de oncológica con los márgenes de seguridad correspondientes, por lo que en la actualidad sigue siendo un desafío para los especialistas elegir la técnica más adecuada en cada caso.

Como premisas en la reconstrucción auricular debemos tener en cuenta la simetría, la implantación, el tamaño y el mantenimiento del armazón cartilaginoso junto con la cobertura cutánea ${ }^{6}$.

Desde hace décadas, las técnicas más utilizadas para la reconstrucción del hélix han sido la resección simple en cuña para lesiones de hasta $1,5 \mathrm{~cm}$ y las exéresis en estrella, semiluna o en copa para defectos de hasta 2.5 $\mathrm{cm}$; con resultados aceptables. Sin embargo estas técnicas pueden causar deformidades o escotaduras en el hélix con un resultado inestético evidente.

Por otra parte, la resección en cuña escalonada permite una distribución de las fuerzas elásticas más uniforme y disminuye la tensión que sufren los tejidos. De esta manera el diseño del triángulo de compensación escalonado tendrá como consecuencia directa la cicatrización con mínimas complicaciones y un resultado estético superior al resto de las técnicas. Cabe agregar que 

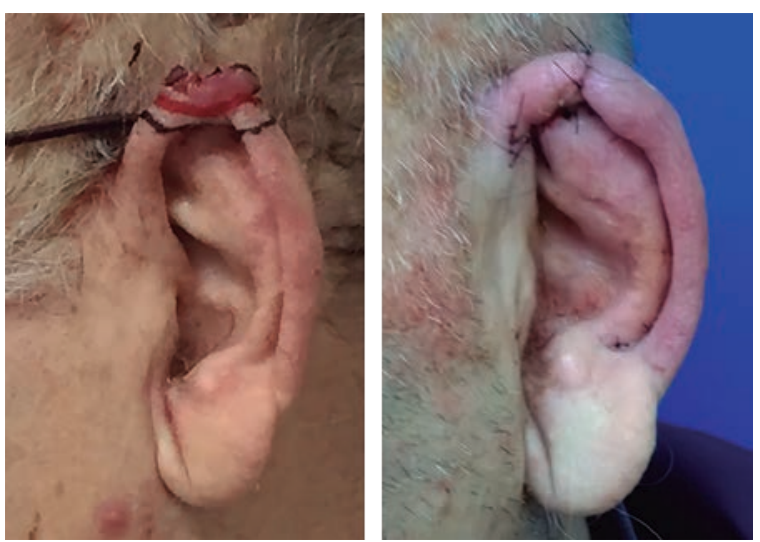

Figura 3. Colgajo de Antia y Buch.

la técnica mencionada tiene indicación en defectos de hasta $2,5 \mathrm{~cm}$ de espesor total ubicadas en el hélix ${ }^{4,6,7}$.

Con respecto a la técnica de Antia y Buch, la misma tiene indicación en lesiones de espesor total del hélix de hasta $3 \mathrm{~cm}$ de longitud, ya que en defectos mayores la asimetría con la oreja contralateral se torna muy evidente. Tiene la gran ventaja de mantener la anatomía normal auricular pero solo con disminución leve del tamaño. Cuando la asimetría es notoria se puede realizar la plastia la oreja normal ${ }^{2,6,7}$.

\section{CONCLUSIÓN}

Actualmente no hay un umbral definido para la elección de cada técnica. Creemos que, para lesiones menores de $3,5 \mathrm{~cm}$ de espesor total en el hélix, del tercio medio y superior auricular, se puede indicar la técnica de Antia y Buch; y para defectos menores a $2,5 \mathrm{~cm}$ la de cuña escalonada. Para orejas grandes preferimos Antia y Buch y para pequeñas con mayor tensión cutánea a la hora de la resección, la de cuña escalonada.

\section{BIBLIOGRAFÍA}

1. Brent B. Reconstruction of the Auricle. In: Mc Carthy JG (Ed), Plastic Surgery. Philadelphia: W. B. Saunders Co., 1990. Vol 3, Ch 40, 2094-2152.

2. Antia NH, Buch VI. Chondrocutaneous advancement flap for the marginal defect of the ear. Plast Reconstr Surg 1967:39:472.

3. Radonich M, Zaher E. Auricular reconstruction of helical rim defects: wedge resection revisited. Dermatol Surg 2002;28:62-5.

4. Mahajan AL. Helix reconstruction with wedge resection in the right place. J Plast Reconstr Aesthet Surg 2009;62:125-7.
5. Mohs FE. Mohs Micrographic surgery. A historical perspective. Dermatol clin 1989;7:609-11.

6. Garcia M. Atlas de colgajos locales en la reconstrucción de cabeza y cuello. Cap. 11, págs. 327-49. Ed Elsevier, 2007, Madrid, España.

7. McCarthy, Galiano, Boutros; Current Therapy in Plastic Surgery; Reconstruction of the auricle; 2006. 